IER3IP1， LMNA， NEUROG3， PAX6， PLAGL1， SLC19A2, SLC2A2, SH2B1, SERPINB4, MADD).

Results Overall, 53 out of 99 patients (53.5\%) had genetic variants in the target genes. The most common mutations in the group of patients with MODY were mutations in the GCK gene $-80 \%(\mathrm{n}=36)$, HNF1A 13.3\% $(\mathrm{n}=6)$, WFS1 $4.4 \%$ $(\mathrm{n}=2)$, PAX4 $=2.2 \%(\mathrm{n}=1)$.

In cases of neonatal diabetes, including genetic syndromes with diabetes, significant mutations were detected in 8 of 18 patients. Thanks to the possibility to performed whole-exome sequencing, we managed to identify and genetically confirm rare syndromic forms of diabetes, such as: diabetes in the structure of Wolcott-Rallison syndrome (mutation in the EIF2AK3 gene) $(n=1)$, diabetes in the structure of IPEX syndrome $(n=1)$, as well as diabetes due to a mutation in the GATA6 gene $(\mathrm{n}=1)$ and a biallelic mutation in the SLC19A2 gene $(n=1)$.

Conclusions Using basic differential diagnostic criteria to establish monogenic diabetes, the molecular genetic confirmation of the diagnosis among suspected patients amounts to $53 \%$. Higher mutation detection rate may be achieved by increasing number of genes tested. One more advantage of whole-exome sequencing should be mentioned: DNA sequencing data may be easily stored for further analysis of newly discovered candidate genes.

\section{GP135 PREVALENCE OF OVERWEIGHT AND OBESITY IN CHILDREN WITH TYPE 1 DIABETES ATTENDING A TERTIARY CLINIC IN IRELAND AND THE RELATIONSHIP WITH GLYCAEMIC CONTROL}

${ }^{1}$ Eadaoin Hayes* ${ }^{1,2}$ Cody Declan. ${ }^{1}$ National Children's Research Centre, Dublin, Ireland; ${ }^{2}$ Our Lady's Children's Hospital Crumlin, Dublin, Ireland

\subsection{6/archdischild-2019-epa.199}

Aims We aim to find the prevalence of overweight and obesity in children aged over the age 5 with type 1 diabetes who attend the tertiary referral clinic in our Lady's Children's Hospital Crumlin, Dublin.

Methods We measured heights and weights of the children with type 1 diabetes attending the clinic. We entered the data into our electronic database (Diamond version 1, Hicom). The electronic database calculates the child's body mass index $(\mathrm{kg} /$ $\mathrm{m}^{2}$ ). We exported the data to SPSS Version 24 IBM. We calculated the children's BMI Z-score using the WHO Reference 2007 SPSS macros package. We used the WHO 2007 Reference normative data and the definition of overweight as BMI z-score for age more than 1 standard deviation away from the mean (represents $85^{\text {th }}$ centile). We used the WHO definition of obesity as BMI z-score for age of more than 2 standard deviations away from the mean (represents $97^{\text {th }}$ centile). We ran descriptive statistics. We analysed the relationship between BMI z-score and HbA1c using a linear regression model.

Results 541 children attended the diabetes clinic. 511 of these children were over the age of 5 . Of these children $38.7 \%$ had a BMI $z$-score +1 SD away from the mean in the WHO reference data reflecting overweight. Of the 511 children 47 or $9.2 \%$ of them had a BMI $\mathrm{z}$-score $+2 \mathrm{SD}$ from the mean normative data reflecting obesity and $1.2 \%$ had a BMI z-score of $+3 \mathrm{SD}$ away from the mean reflecting severe obesity. There was no statistically significant relationship between BMI zscore and HbA1c using linear regression.
Conclusion Our results highlight the high prevalence of overweight and obesity in children with type 1 diabetes. The prevalence of overweight and obesity is nearly double that of the general population. This is a relatively new phenomena. Various causes have been postulated including intensive insulin therapy since the early $90 \mathrm{~s}$ as well as the secular trend in overweight and obesity.

\section{GP136 PSYCHOSOCIAL RISK ASSESSMENT IN CHILDREN WITH TYPE 1 DIABETES IN IRELAND}

${ }^{1,2}$ Elena Hennessy* ${ }^{*}{ }^{1}$ Conor Cronin, ${ }^{1}$ Anne Bradfield, ${ }^{1}$ Pankaj Agrawal, ${ }^{3}$ Orla Neylon, ${ }^{4}$ Akhtar Khan, ${ }^{2}$ Patricia Leahy, ${ }^{1}$ Susan O'Çonnell, ${ }^{1,2}$ Stephen MP O'Riordan. ' Cork Unversity Hospital, Cork, Ireland; ' University College Cork, Cork, Ireland; ${ }^{3}$ University Hospital Limerick, Limerick, Ireland; ${ }^{4}$ Tralee General Hospital, Tralee, Ireland

\subsection{6/archdischild-2019-epa.200}

Objectives To evaluate and compare the risk for poor glycaemic control at two time points in an Irish cohort of children with T1D.

Methods The Risk Index for Poor Glycaemic Control (RIPCG) is the screening tool to assess psychosocial risk where each score increases the risk of poor control and DKA on $10 \%$ (low risk score $0-1$, moderate $=2$, high risk $\geq 3$ ). The baseline data was collected for 2 years while follow up data collection (T1) began at least 6 months after the start of the study and continued for 2 years. No intervention was involved.

Results As a part of 2-year longitudinal study 245 children with T1D (129 males) aged 3-18 years (mean 11.7 \pm 3.5 ) were analysed at baseline. Total of 90 patients out of 245 were assessed for psychosocial risk at baseline and at T1 (Table 1).

Abstract GP136 Table 1 Percentage of patients at low, moderate and high risk on RIPGC at baseline and at T1

\begin{tabular}{lll}
\hline Risk category & Baseline & T1 \\
\hline Low & $68.8 \%$ & $73.8 \%$ \\
Moderate & $16.3 \%$ & $11.2 \%$ \\
High & $15 \%$ & $15 \%$ \\
\hline
\end{tabular}

At baseline: $31.3 \%$ of patients had a moderate score and high scores on RIPGC. At T1: $26.2 \%$ of patients had a moderate score and high psychosocial risk scores. Paired analysis showed that the difference in RIPGC score between baseline and T1 was not significant $(\mathrm{p}>0.05)$. Three patients $(3.3 \%)$ increased the risk from low to moderate, another 3 patients (3.3\%) from low to high risk and 2 patients (2.2\%) from moderate to high risk. However, 12 patients $(13.3 \%)$ reduced the risk with a time: 7 patients $(7.7 \%)$ moved from category of moderate risk to low risk, $3(3.3 \%)$ - from high to low and $2(2.2 \%)$ - from high to moderate risk category. The distribution of low, moderate and high risk patients did not differ significantly in baseline group and T1 $(p>0.05)$

Conclusions Almost one third of children with T1D in Irish population are at moderate and high psychosocial risk. The routine care provided by health professionals doesn't reduce this risk significantly with time. Our data indicates the need of intervention by trained clinical psychologist for children with T1D and psychosocial risk. 